\title{
Does Nasal Septal Deviation May Have an Influence on Distribution Patterns of the Frontal Ethmoidal Cells?

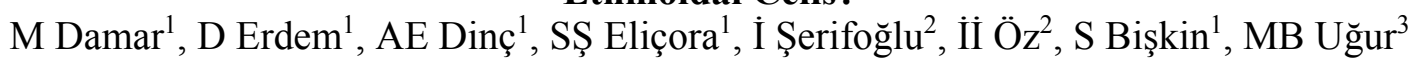

\begin{abstract}
Objective: To investigate the role of nasal septal deviation on the presence or distribution patterns of the frontal ethmoidal cells (types I to IV), the pneumatization patterns of the frontal sinus, and to search the association between the frontal ethmoidal cells and the pneumatization degree of the frontal sinus and the presence of concha bullosa.
\end{abstract}

Methods: Paranasal sinus computed tomography scans of 268 patients with nasal septum deviation (178 males, 90 females; age range 18-65 years) were retrospectively evaluated for the presence and distribution patterns of frontal ethmoidal cells, agger nasi cells, concha bullosa, and the pneumatization degree of the frontal sinus on the deviated side and contralateral side.

Results: The nasal septum was deviated to the right in 135 patients and to the left in 133 patients. There was no significant difference in terms of presence of agger nasi cells, pneumatization degrees of the frontal sinuses, and frontal ethmoidal cells and at the ipsilateral (deviated side) and contralateral sides. When there was a concha bullosa, there were significantly more frontal ethmoidal cells on the ipsilateral side but not on the contralateral side.

Conclusion: Our study suggests that the presence and distribution of frontal ethmoidal cells are not influenced by nasal septal deviation.

Keywords: Frontal ethmoidal cell, Nasal septal deviation, Concha bullosa, Frontal sinus

From: ${ }^{1}$ Department of Otorhinolaryngology Head and Neck Surgery, Bülent Ecevit University Faculty of Medicine. Zonguldak, Turkey. ${ }^{2}$ Department of Radiology, Bülent Ecevit University Faculty of Medicine. Zonguldak, Turkey. ${ }^{3}$ Department of Otorhinolaryngology Head and Neck Surgery, Gazi University Faculty of Medicine. Bülent Ecevit University Hospital, Zonguldak/ Turkey.

Correspondence: Dr M Damar, Department of Otolaryngology, Faculty of Medicine, Bulent Ecevit University, Kozlu/Zonguldak, 67600, Turkey. Fax: +90 (372) 2610264, e-mail: drmuratdamar@yahoo.com 


\section{INTRODUCTION}

Endoscopic sinus surgery is one of the common procedures performed by otolaryngologists today. Through wide usage of this surgery anatomy and radiology of the nose and the paranasal sinuses is better understood. But the surgery of the frontal sinus still remains challenging and is a cause of failure for surgeons $(1,2)$. The hidden location of the frontal recess, the anatomical variations of pneumatization patterns and the important proximity with vital structures such as the anterior skull base, the orbit and the lacrimal region make this area inaccessible and thus incomplete removal of the cells usually causes recurrence of the disease $(2-4)$.

New developments in imaging technologies, especially usage of high definition spiral multi-slice computed tomography (CT) scanners in axial, coronal and sagittal planes, make these variable cellular patterns easier to be understood (4). According to the CT data, the ethmoid cells were re-classified by many authors $(4,5)$. Agger nasi cells (ANCs), frontal ethmoidal cell types I to IV (FECs), suprabullar cells (SBCs), frontal bullar cells (FBCs), supraorbital ethmoid cells (SOECs) and interfrontal sinus septal cells (IFSSCs) are well defined ethmoid cells that build the frontal recess. Although variable patterns of the FECs are well understood, no publication still emphasizes why in some patients some cells are present and in others not, or why the distribution patterns are so different between individuals.

Importance of available nasal airflow and normal oxygen pressure on the nasal mucosa for the development of paranasal sinuses has been demonstrated by previous studies $(6,7)$. Nasal septal deviation (NSD) is a common anatomical deformity and one of the most important reasons that disrupts normal nasal physiology. It is involved in almost all rhinologic problems to some degree and it may affect the development of the paranasal sinuses. In addition, NSD is frequently encountered in patients undergoing endoscopic sinus surgery and its effect on the anatomy of the frontal sinus needs to be clarified. However, the effect of NSD to the 
pneumatization patterns of the frontal recess and the presence of the FECs was not investigated before in the literature.

The aim of this study is to investigate the role of nasal septal deviation (NSD) on the presence and the distribution patterns of the FECs (types I to IV), and on the pneumatization patterns of the frontal sinus; and to search the association between FECs and the pneumatization degree of the frontal sinus and the presence of concha bullosa (CB).

\section{METHODS}

\section{Patients}

The study was approved by the institutional review board of the Medical School of Bulent Ecevit University. CT images, demographic and clinical characteristics of the patients, were obtained from the archives of Bulent Ecevit University Faculty of Medicine, and analyzed for this retrospective study.

Only patients over 18 years of age were included. Patients with a history of previous nasal trauma or surgery, sinonasal tumors, sinonasal polyposis, inflammatory disease of the frontal recess, fibro-osseous lesions, congenital anomalies, and cerebrospinal fluid leak were excluded from the study.

304 patient who underwent CT examination and septoplasty due to nasal septal deviation were retrospectively identified from January 2013 to June 2015 in our clinic. Paranasal sinus CT had been performed to investigate the presence of any accompanying paranasal sinus pathologies associated with nasal obstruction. CT images of 268 consecutive patients were available for this study. 


\section{CT imaging}

CT examinations were performed by Activion 16 CT Scanner (Toshiba Medical Systems, 2008 Japan). The routine paranasal sinus CT protocol was used for all patients with $120 \mathrm{kVp}$; $100-150 \mathrm{~mA} ; 1.0 \mathrm{~mm}$ or less contiguous axial slice thickness; 512 x 512 matrix size; $240 \mathrm{~mm}$ field of view. Reconstructed coronal and sagittal images were generated using the software performed at a separate Workstation (INFINITT Pacs version 3.0.9.1 BN6).

Firstly the angle of nasal septal deviation (ASD) for each patient was measured on coronal CT scan where the nasal septum was most severely deviated in the region of the ostiomeatal complex (Table 1). The direction of the deviation was described by the convexity of the septal curvature.

We evaluated the images for the presence of $\mathrm{CB}$ and ANCs, the pneumatization degrees of the frontal sinuses (Table 1). The frontal sinus extending across more than onethird of the corresponding orbit was accepted as "good-pneumatized" frontal sinus. The presence and distribution of FECs (type I-IV) on the right and left sides and on the deviation side and contralateral side were determined (Table 1) (Fig. 1-4). ANCs and FECs were determined by magnification and appropriate window setting of the coronal, and sagittal sections. The presence and the distribution of the FECs were determined according to "Modified Kuhn Classification" (9, 20). In this classification, only type IV cell was different from the original one and defined as "a cell pneumatizing through into the frontal sinus and extending $>50 \%$ of the vertical height of the frontal sinus" (Fig. 4) (20). CB was defined as the pneumatization of the most inferior aspect of the middle turbinate (12).

The CT examinations were simultaneously reviewed by a radiologist and an otolaryngologist (I. S. and M.D.) and differences in opinions were resolved by consensus. 


\section{Statistical analysis}

Statistical analyses were performed with SPSS 19.0 software (SPSS Inc., Chicago, IL, USA). Distribution of data was determined by Shapiro-Wilk test. Continuous variables were expressed as mean \pm std. deviation and categorical variables as frequency and percent. Continuous variables were compared with the Mann-Whitney U test. Mc Nemar and Marginal homogeneity tests were used for related measures. Kruskal-Wallis test was used to determine association between the pneumatization degree of the frontal sinus and frontal ethmoidal cells. The Dunn's test was used for post-hoc test after Kruskal-Wallis test. $P$ value of less than 0.05 was considered statistically significant for all tests.

\section{RESULTS}

A total of 268 patients consisting of 135 right-sided and 133 left-sided septal deviation deformities were included in the study. There were 178 males and 90 female patients. The mean age was $36.5 \pm 12.5$ years (range: 18 to 65 years). The measured angles of nasal septal deviations were found to range between 5 and 21.5 degrees (mean $10.1 \pm 3.8$ degrees). Deviation angles were $10.2 \pm 4.1$ degrees for the right deviation and $10.0 \pm 3.4$ degrees for the left deviation. There was no statistically significant difference among the deviation angles between the right and left deviations $(P=0.762)$.

The percentage and the prevalence of ANCs, FECs, CB, and the pneumatization degrees of the frontal sinus identified on CT for right and left sides are shown in Table 2. ANCs were present in 241 patients $(89.9 \%)$ on the right and 249 patients $(92.9 \%)$ on the left side. The ratio of good-pneumatized frontal sinuses were $82.1 \%$ on the right side and $82.5 \%$ on the left side. CB was found in 66 of 268 patients (24.6\%) on the right side and in 56 of 268 patients $(20.9 \%)$ on the left side. When we evaluated the presence of FECs, we identified 
FECs in 94 of 268 patients (35.1\%) on the right side, in 97 of 268 patients $(36.2 \%)$ on the left side. There were no significant differences in terms of these parameters between the right and the left sides $(P>0.05)$.

The comparison of the percentage and the prevalence of ANCs, various FECs, CB and the pneumatization degrees of the frontal sinus at the ipsilateral side (deviated side) and contralateral side are given in Table 3. ANCs were found in 244 patients $(91 \%)$ on the ipsilateral side and 245 patients $(91.4 \%)$ on the contralateral side. The ratio of goodpneumatized frontal sinuses were found $82.4 \%$ at the ipsilateral side and $82.1 \%$ at the contralateral side. We identified FECs in 99 of 268 patients (36.9\%) on ipsilateral side and in 91 of 268 patients $(34 \%)$ on contralateral side. No difference was found among these parameters according to the septal deviation side $(P>0.05)$. However, we found $\mathrm{CB}$ in 41 of 268 patients $(15.3 \%)$ on the ipsilateral side and in 81 of 268 patients $(30.2 \%)$ on contralateral side. This difference was statistically significant $(P<0.001)$.

There was also a statistically significant relationship between the pneumatization degrees of the ipsilateral frontal sinus and the presence of ipsilateral frontal ethmoidal cell type III and type IV and the pneumatization degrees of the contralateral frontal sinus and the presence of contralateral frontal ethmoidal cell type III and type IV ( $P=0.026, P=0.003$, respectively) (Fig. 5). When there was a $\mathrm{CB}$ at the ipsilateral side of the septal deviation, the presence of ipsilateral FECs was increased by the presence of $\mathrm{CB}$ and that was also found to be statistically significant $(P<0.001)$. However, there was no significant difference in terms of presence of the FECs on the contralateral side $(P=0.382)$ (Fig. 6). 


\section{DISCUSSION}

Endoscopic sinus surgery is currently accepted as the treatment choice for chronic sinusitis resistant to medical treatment $(14,15)$. Widespread usage of this surgery has led a better understanding of the anatomy of the paranasal sinuses. Despite the most sophisticated radiologic techniques and surgical tools, the frontal sinus and frontal recess still remains one of the most complex anatomic areas of the anterior skull base, with many anatomic variations $(2,4,11)$. A thorough knowledge of the common anatomic variants is essential to avoid complications and failure during surgery $(4,14)$. The small diameter, the anterior location, and orientation within the frontal bone as well as the anatomic relationship with the orbit, skull base, and different groups of frontal recess cells make the anatomy difficult to identify (4).

The amount of the nasal air space influences the amount of positive pressure on the nasal mucosa and nasal airflow. One of the most important causes that affect nasal airflow is NSD. The effects of NSD to the development of paranasal sinuses and adjacent anatomical structures have been investigated before in the literature. Orhan et al. demonstrated that in adult patients with septal deviation, maxillary sinus volume was smaller on the deviation side (16). On another study, severe septal deviation on the ipsilateral side had been shown significantly reduce the mastoid air volume (17). Sazlisoy et al found that cribriform plate depth at the contralateral side was significantly higher than that of the ipsilateral side in adult patients with NSD (18). Karataş et al. demonstrated that there was no effect of NSD on frontal sinus volume (19). In present study, we found that there was no difference between ipsilateral and contralateral sides in patients with NSD in term of the pneumatization of the frontal sinuses $(P=0.252)$.

Bent and Kuhn, classified the frontal infundibular air cells into 4 types (5). These cells develop clinical significance either by obstructing the frontal recess or by becoming primarily 
infected. A review of the literature suggests that FECs show a broad spectrum of prevalence $(2,4,7)$. FECs have been reported in $20 \%$ to $41 \%$ incidence of sinus specimens $(2,20)$. We identified FECs in $36.9 \%$ on ipsilateral side and $34 \%$ on contralateral side, this difference was not statistically significant $(P=0.292)$.

There are a few studies that investigate factors affecting the distribution patterns of FECs. These studies have shown that the presence of FECs is positively associated with hyperpneumatization of the frontal sinus and the presence of bullous concha bullosa $(2,21)$. We also found that there was a statistically significant relationship between the pneumatization degrees of the frontal sinus and the presence of frontal ethmoidal cell type III and type IV at ipsilateral and contralateral side $(P<0.05)$. In the present study, there was a significant difference between ipsilateral and contralateral sides in terms of concha bullosa $(P$ $<0.001)$. At the same time, we also found that when there was a concha bullosa on the ipsilateral side of the septal deviation, the presence of ipsilateral FECs was increased by the presence of concha bullosa and that was also found to be statistically significant $(P<0.001)$. However, there was no significant difference in terms of presence of the FECs on the contralateral side $(P=0.382)$.

Our study has some limitations. Evaluation of CT images for the presence of various cells is not exactly an objective method and evaluation may vary among individuals. To minimize the mistakes, CT examinations were simultaneously reviewed by a radiologist (Dr. Serifoglu) and an otolaryngologist (Dr. Damar), and any differences in opinions were resolved by consensus. Nevertheless the studied radiologic images are not the actual anatomy, the gold standard for a study of prevalence like this would be a cadaver dissection combined with CT scanning in multiple planes (2). 


\section{CONCLUSIONS}

Our study suggests that the presence and distribution of FECs and the pneumatization of the frontal sinuses is independent from NSD. According to our study we can say that there is another unknown parameter or mechanism rather than septal deviation that influences the pneumatization patterns of FECs. However, FECs are associated with other variants of pneumatization. If a surgeon is planning frontal recess surgery due to frontal sinusitis, the presence of FECs should be taken into account during the endoscopic sinus surgery in the presence of ipsilateral $\mathrm{CB}$ or the hyperpneumatization of the frontal sinuses determined by preoperative CT imaging.

\section{ACKNOWLEDGEMENTS}

Conflict of Interest: None of the authors has any conflicts of interest with regard to this study. 


\section{REFERENCES}

1. Han D, Zhang L, Ge W, Tao J, Xian J, Zhou B. Multiplanar computed tomographic analysis of the frontal recess region in Chinese subjects without frontal sinus disease symptoms. ORL 2008; 70: 104-12.

2. Meyer TK, Kocak M, Smith MM, Smith TL. Coronal computed tomography analysis of frontal cells. Am J Rhinol 2003; 17: 163-68.

3. Cho JH, Citardi MJ, Lee WT, Sautter NB, Lee HM, Yoon JH, et al. Comparison of frontal pneumatization patterns between Koreans and Caucasians. Otolaryngol Head Neck Surg 2006; 135: 780-88.

4. Tuncyurek O, Songu M, Adibelli ZH, Onal K. Frontal infundibular cells: Pathway to the frontal sinus. Ear Nose Throat J 2012; 91: 29-32.

5. Bent JP, Cuilty-Siller C, Kuhn FA. The frontal cell as a cause of frontal sinus obstruction. Am J Rhinol 1994; 8: 185-91.

6. Kossowska E, Gasik C. Results of surgical treatment of choanal atresia. Rhinology 1979; 17: 155-60.

7. Parsons DS, Wald ER. Otitis media and sinusitis: similar diseases. Otolaryngol Clin North Am 1996; 29: 11-25.

8. Chaiyasate S, Baron I, Clement P. Analysis of paranasal sinus development and anatomical variations: a CT genetic study in twins. Clin Otolaryngol 2007; 32: 93-7.

9. McLaughlin RB, Rehl RM., Lanza DC. Clinically relevant frontal sinus anatomy and physiology. Otolaryngol Clin North Am 2001; 34: 1-22.

10. Kuhn FA. Chronic frontal sinusitis: the endoscopic frontal recess approach. Operative techniques Otolaryngol Head Neck Surg 1996; 7: 222-9.

11. Wormald PJ. Surgery of the frontal recess and frontal sinus. Rhinology 2005; 43(2): $82-85$. 
12. Wormald PJ. The axillary flap approach to the frontal recess. Laryngoscope 2002; 112: 494-9.

13. Wormald PJ, Chan SZX. Surgical techniques for the removal of frontal recess cells obstructing the frontal ostium. Am J Rhinol 2003; 17: 221-6.

14. Lien CF, Weng HH, Chang YC, Lin YC, Wang WH. Computed tomographic analysis of frontal recess anatomy and its effect on the development of frontal sinusitis. Laryngoscope $2010 ; 120: 2521-7$.

15. Wormald PJ. The agger nasi cell: The key to understanding the anatomy of the frontal recess. Otolaryngol Head Neck Surg 2003; 129: 497-107.

16. Orhan I, Ormeci T, Aydın S, Altin G, Urger E, Soylu E, Yilmaz F. Morphometric analysis of the maxillary sinus in patients with nasal septum deviation. Eur Arch Otorhinolaryngol 2014; 271: 727-32.

17. Gencer ZK, Ozkiris M, Okur A, Karacavus S, Saydam L. The possible associations of septal deviation on mastoid pneumatization and chronic otitis. Otol Neurotol 2013; 34: $1052-7$.

18. Saylisoy S, Acar M, San T, Karabag A, Bayar Muluk N, Cingi C. Is there a relationship between cribriform plate dimensions and septal deviation angle? Eur Arch Otorhinolaryngol 2014; 271: 1067-71.

19. Karatas D, Koc A, Yüksel F, Doğan M, Bayram A, Cihan MC. The effect of nasal septal deviation on frontal and maxillary sinus volumes and development of sinusitis. $\mathbf{J}$ Craniofac Surg 2015; 26: 1508-12.

20. DelGaudio JM, Hudgins PA. Multiplanar computed tomographic analysis of frontal recess cells. Arch Otolaryngol Head Neck Surg 2005; 131: 230-5.

21. Yildirım A. Is it more reasonable to categorize frontal cells on the basis of their location rather than on their type? Ear Nose Throat J 2010; 89: 19-21. 
Table 1: Evaluated parameters on computed tomography

Frontal sinus pneumatization: Lateral extension of the frontal sinus (8).

* 0 No pneumatization

* 1 Less than one-third of the corresponding orbit

* 2 One-third to two-third of the corresponding orbit

* 3 Over two-thirds of the corresponding orbit

Agger nasi cell: Pneumatization immediately anterior and superior to insertion of the middle turbinate (9).

Concha bullosa: Pneumatization of the inferior most aspect of the middle turbinate (2).

Modified Kuhn Classification of frontal ethmoidal cells $(10,11)$

* Type 1 Single frontal recess cell above agger nasi cell (Figure 1)

* Type 2 Tier of cells in frontal recess above agger nasi cell (Figure 2)

* Type 3 Single massive cell pneumatizing cephalad into frontal sinus (Figure 3)

* Type 4 (modified from original classification A cell pneumatizing through into the frontal sinus and extending $>50 \%$ of the vertical height of the frontal sinus (Figure 4) $(11,12,13)$

The angle of septal deviation (ASD): The angle is defined as the angle between a line drawn from the superior insertion of the septum at the crista galli to the inferior insertion of the septum at the level maxillary crest and another line from the superior insertion of the septum at the crista galli to the apex of the septal deviation in the region of the osteomeatal complex. 
Table 2: The percentage and prevalence of ANCs, FECs, CB, and the pneumatization degrees of frontal sinuses identified on $\mathrm{CT}$ for the right and left sides.

\begin{tabular}{llll}
\hline Cell types & Right side (N, \%) & Left side (N, \%) & P \\
\hline Agger nasi cell (ANCs) & $27(10.1 \%)$ & $19(7.1 \%)$ & 0.134 \\
No & $241(89.9 \%)$ & $249(92.9 \%)$ & \\
Available & & & \\
Frontal ethmoidal cells (FECs) & $174(64.9 \%)$ & $171(63.8 \%)$ & \\
No & $55(20.5 \%)$ & $45(16.8 \%)$ & \\
Type 1 & $8(3 \%)$ & $7(2.6 \%)$ & \\
Type 2 & $28(10.4 \%)$ & $36(13.4 \%)$ & \\
Type 3 & $3(1.1 \%)$ & $9(1.1 \%)$ & \\
Type 4 & & & \\
Concha bullosa (CB) & $202(75.4 \%)$ & $212(79.1 \%)$ & \\
No & $66(24.6 \%)$ & $56(20.9)$ & \\
Available & & & \\
The pneumatization degrees & & & \\
sinuses & frontal & $4(1.5)$ & \\
0 & $4(1.5 \%)$ & $43(16 \%)$ & \\
1 & $44(16.4 \%)$ & $102(38.1)$ & \\
2 & $119(44.4 \%)$ & $119(44.4 \%)$ & \\
3 & $101(37.7 \%)$ & & \\
\hline
\end{tabular}

$\mathrm{N}=$ the number of patients. 
Table 3: The comparison of percentage and prevalence of ANCs, FECs, CB, and the pneumatization degrees of frontal sinus at the ipsilateral (deviated side) and contralateral sides identified on computed tomography.

\begin{tabular}{llll}
\hline Cell types & Ipsilateral side (N, \%) & Contralateral side (N, \%) & P \\
\hline Agger nasi cell (ANCs) & & & 1.000 \\
No & $24(9 \%)$ & $23(8.6 \%)$ & \\
Available & $244(91 \%)$ & $245(91.4 \%)$ & 0.292 \\
Frontal ethmoidal cells (FECs) & & & \\
No & $169(63.1 \%)$ & $177(66 \%)$ & \\
Type 1 & $51(19 \%)$ & $49(18.3 \%)$ & \\
Type 2 & $7(2.6 \%)$ & $8(3 \%)$ & \\
Type 3 & $34(12.7 \%)$ & $30(11.2 \%)$ & 0.001 \\
Type 4 & $7(2.6 \%)$ & $4(1.5 \%)$ & 0,252 \\
Concha bullosa (CB) & & & \\
No & $227(84.7 \%)$ & $187(69.8 \%)$ & \\
Available & $41(15.3 \%)$ & $81(30.2 \%)$ & \\
The pneumatization degrees of the frontal & & & \\
sinuses & & $5(1.9)$ & \\
0 & $3(1.1 \%)$ & $43(16 \%)$ & \\
1 & $44(16.4 \%)$ & $115(42.9)$ & \\
2 & $107(39.9 \%)$ & $105(39.2 \%)$ & \\
3 & $114(42.5 \%)$ &
\end{tabular}

$\mathrm{N}=$ the number of patients. 

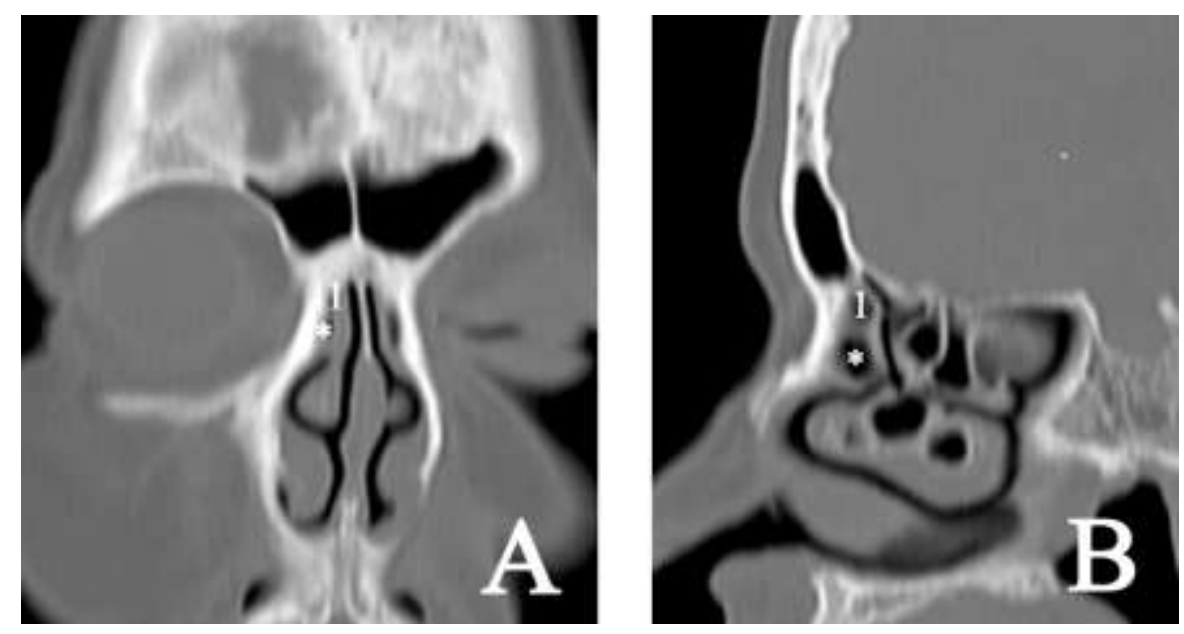

Fig. 1: A patient with left- sided septal deviation has FEC type 1 above agger nasi cell (ANC) (*) on the right side on the coronal (A) and parasagittal (B) CT images.
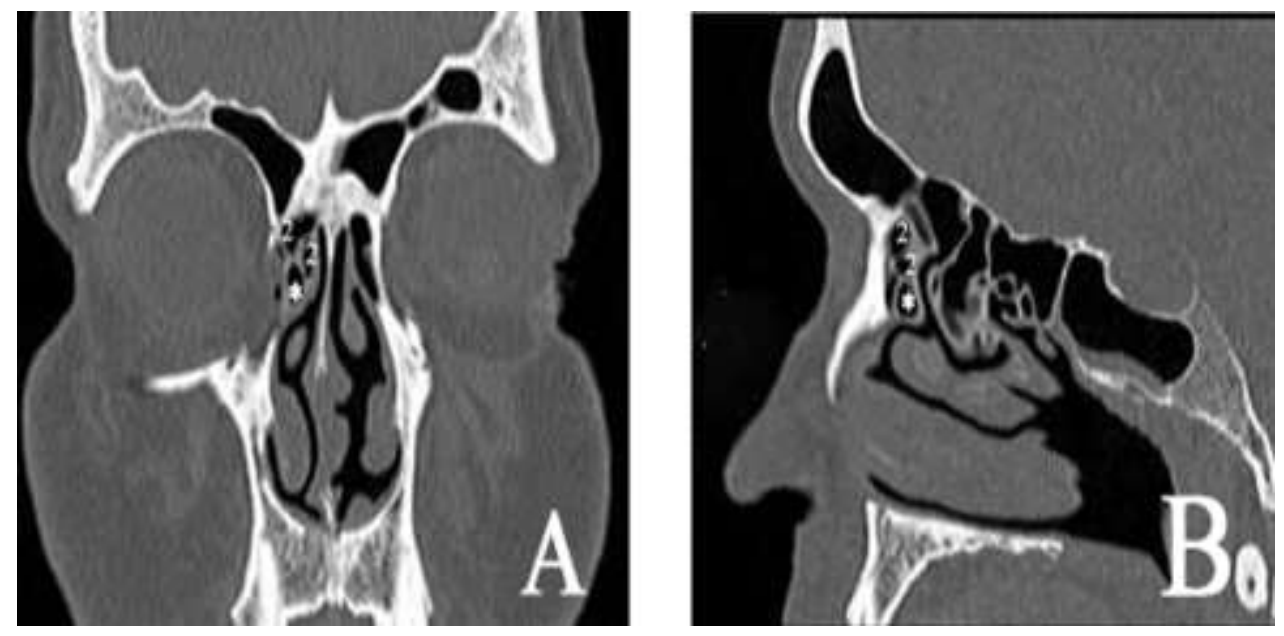

Fig. 2: A patient with right- sided septal deviation has FEC type 2 as tiered cells above ANC (*) on the right side on the coronal (A) and parasagittal (B) CT images. 

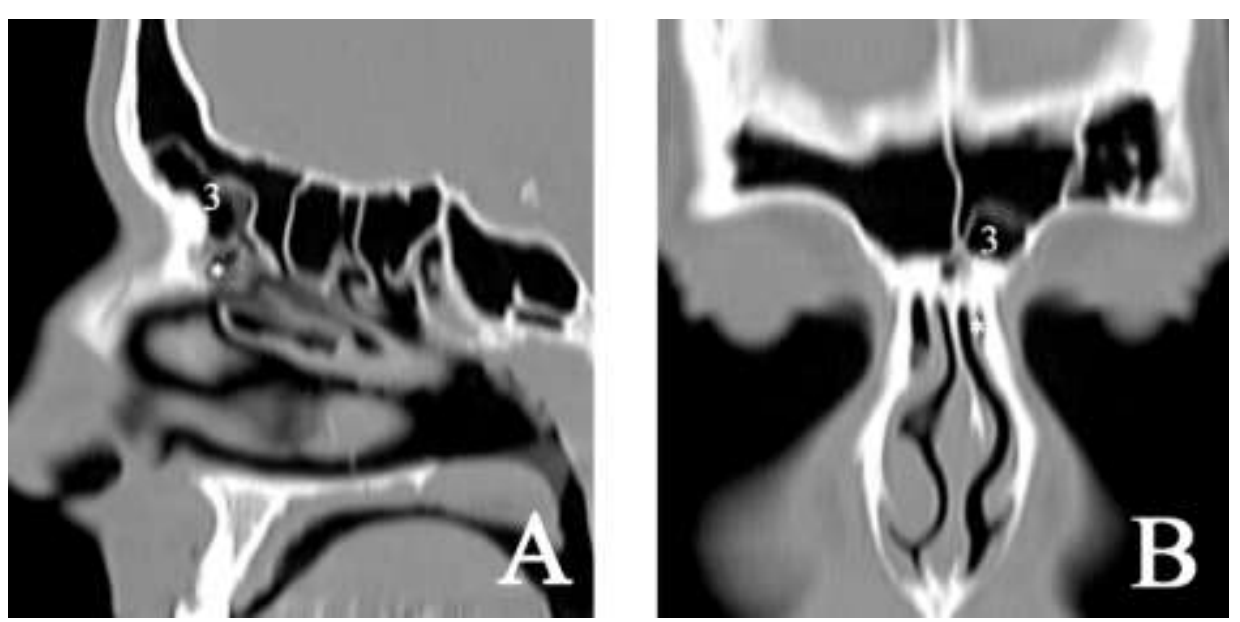

Fig. 3: A patient with left- sided septal deviation has FEC type 3 above ANC (*) and extending into frontal sinus on left side on parasagittal (A) and coronal (B) CT images.
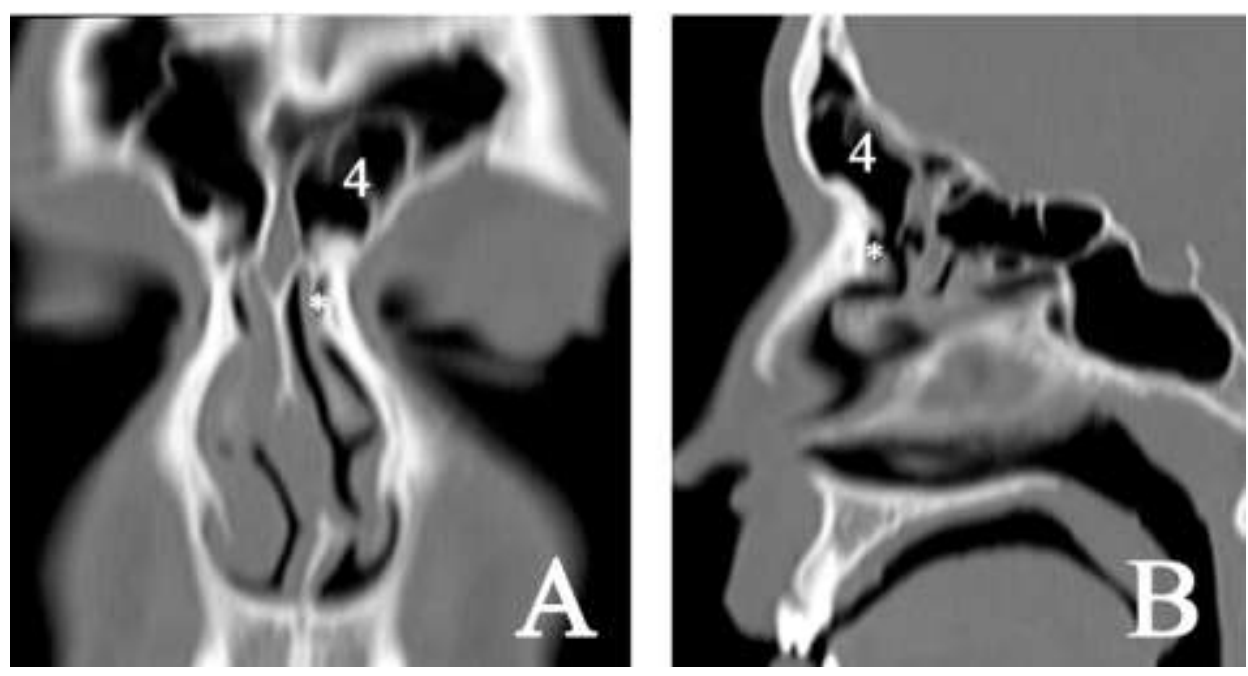

Fig. 4: A patient with left- sided septal deviation has FEC type 4 on the left frontal sinus on the coronal (A) and parasagittal (B) CT images. 

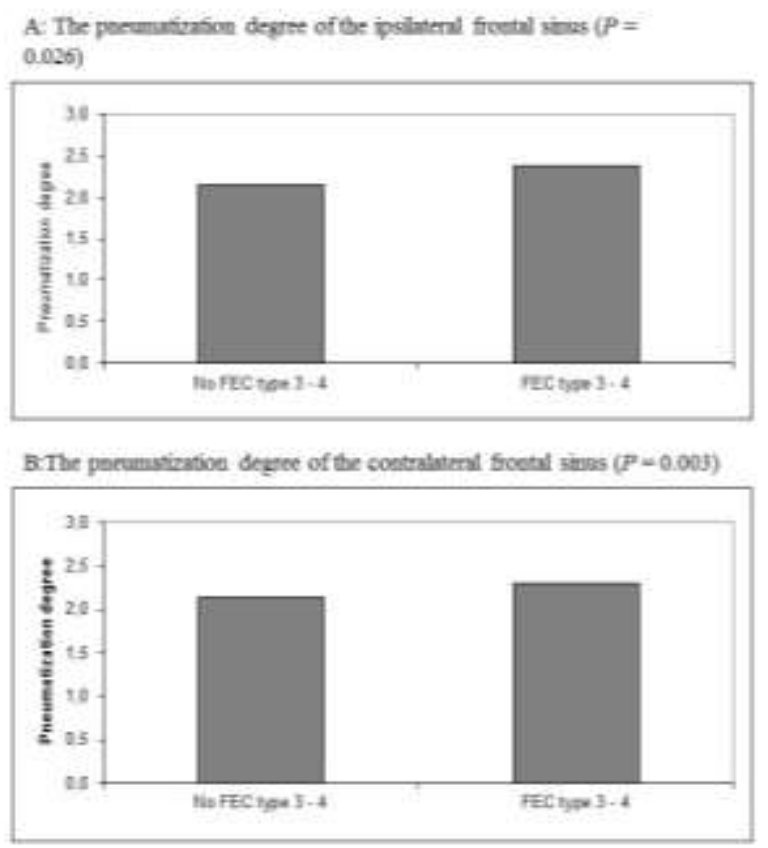

Fig. 5: Association between presence of ipsilateral FEC type 3 - 4 and pneumatization degree of ipsilateral frontal sinus (A), presence of FEC type $3-4$ and pneumatization degree of contralateral side (B).
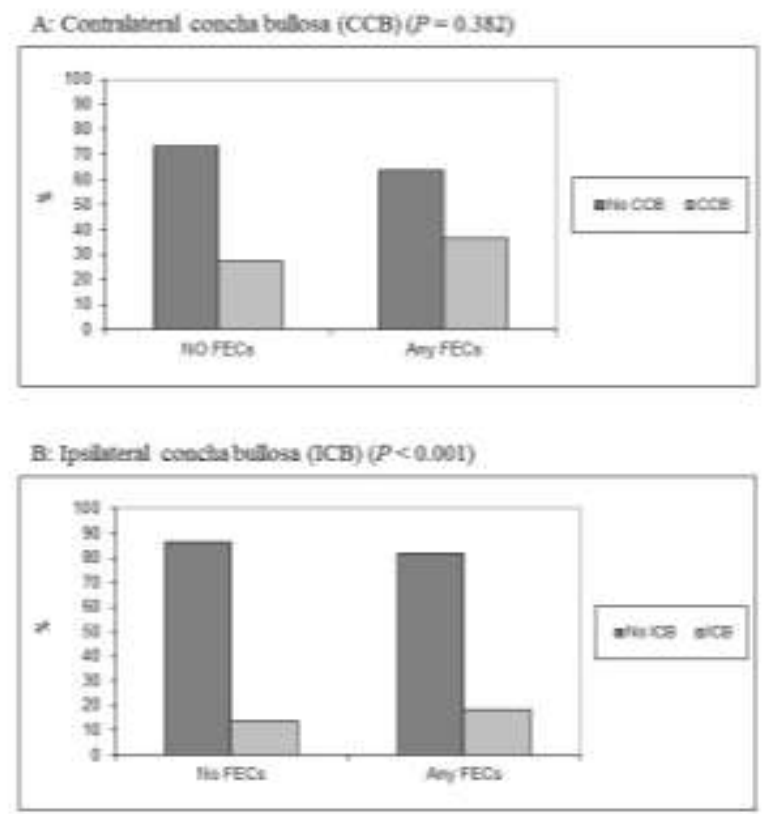

Fig. 6: Association of contralateral $\mathrm{CB}$ and contralateral FECs (A); Ipsilateral $\mathrm{CB}$ and ipsilateral FECs (B). "No FECs" indicates individuals without FECs. Any FECs indicates individuals with any type of FECs. 